\title{
Coulisses
}

Revue de théâtre

15 | Hiver 1997

Varia

\section{Un spectacle sur la ville : Urbs. Impressions à la création en Juin 1996}

Dany Vingler

\section{(2) OpenEdition}

Journals

Édition électronique

URL : http://journals.openedition.org/coulisses/4970

DOI : $10.4000 /$ coulisses.4970

ISSN : 2546-9460

Éditeur

Presses universitaires de Franche-Comté

\section{Édition imprimée}

Date de publication : 1 janvier 1997

Pagination : 7-11

ISSN : 1150-594X

\section{Référence électronique}

Dany Vingler, « Un spectacle sur la ville : Urbs. Impressions à la création en Juin 1996 », Coulisses [En ligne], 15 | Hiver 1997, mis en ligne le 26 avril 2019, consulté le 24 octobre 2019. URL : http:// journals.openedition.org/coulisses/4970 ; DOI : 10.4000/coulisses.4970

Ce document a été généré automatiquement le 24 octobre 2019.

Coulisses 


\title{
Un spectacle sur la ville : Urbs. Impressions à la création en Juin 1996
}

\author{
Dany Vingler
}

Nous remercions La Feuille Théâtre qui nous a autorisé à reproduire ses critiques

Ces textes ont été rédigés à la suite de la représentation donnée par le TU dans le cadre de BVOJ Rencontres Jeune Création par quelques membres de l'équipe critique attachée à l'ensemble de cette manifestation et selon la « règle du jeu » suivante :

Une pièce de théâtre a quatre créateurs : l'auteur - le metteur en scène - l'acteur le spectateur.

Tout ce qui est montré sur scène est signe.

QUAND LE RIDEAU S'OUVRE, TOUT DE SUITE, ON VOIT TOUT.

Nécessité d'une grande disponibilité, d'une grande attention au spectacle en tant que tel : ce qui compte, c'est le travail présenté, ce qu'on voit, ce qu'on entend, ce qu'on sent dans l'instant.

DÉCRIRE EST ESSENTIEL.

Repérer les indices, puis les rapprocher pour trouver la dynamique générale du spectacle.

NE PAS DONNER SON IMPRESSION TROP VITE. NE PAS PORTER UN JUGEMENT TOUT DE SUITE. NE PAS LOUER OU CONDAMNER. 


\section{URBS : Le scénario}

1 Ce n'est pas la ville antique que nous présente Joseph Melcore et les acteurs du Théâtre Universitaire de Franche-Comté; mais la ville d'aujourd'hui en tableaux animés, rythmés.

2 Tout d'abord le conseil d'administration, qui décide de la construction d'un groupe d'immeubles - et ici on ne peut qu'admirer la manière avec laquelle l'édification des nouveaux bâtiments nous est montrée à l'aide de cartons posés sur le sol qui se redressent et constituent un ensemble homogène (mais il y a des laissés pour compte : quelques cartons, symbolisant les anciennes maisons, partent avec leurs précédents occupants, défavorisés par la fortune, vers une lointaine banlieue).

Puis un tableau sur les agences immobilières et leurs publicités plus alléchantes les unes que les autres pour attirer le chaland; suivi de tous les détails relatifs aux règlements de copropriété. Ici une fort jolie image, visuelle : quelques angles, éclairé en rouge des immeubles plongés dans le noir.

Ensuite les problèmes personnels - tous différents - des locataires d'HLM, et finalement un tournoiement effréné où se mêlent locataires et maisons.

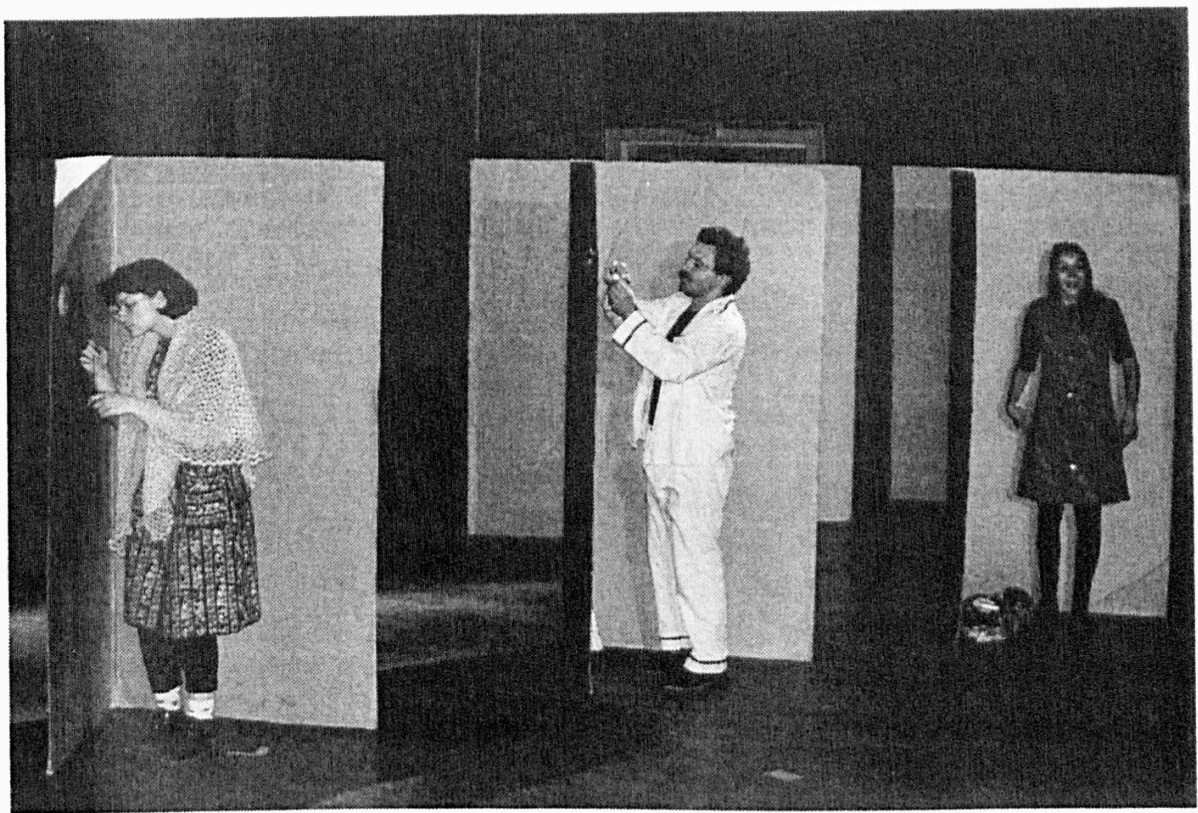

Photo Clément François

4 Mais voici qu'arrive la valse des pancartes de noms de rues, portées par les acteurs en vêtements noirs, qui se croisent, s'éloignent, se rapprochent; ainsi que les trois couleurs des feux de signalisation, qui s'allument de façon rythmique et alternative.

5 Enfin les marginaux de cette société, qui se vengent de ne pouvoir en être, en taggant les murs (ici une idée fort originale de têtes et de bras surgissant des cartons symbolisant les immeubles).

6 Les élus promettent monts et merveilles à leurs futurs électeurs : des zones franches sans impôts, 100000 emplois de ville pour les jeunes, des commerces au cœur de 
quartiers, 4000 policiers supplémentaires, 500000 logements mieux entretenus, etc, etc. On verra bien après les élections s'il sera possible de tenir ces promesses!

Un groupe impressionnant d'architectes futuristes en blouse blanche avec leurs plans blancs sur les bras, prévoyant les mégalopoles de demain (projets qui nous paraissent délirants, mais ont véritablement, nous dit-on, été conçus par des architectes japonais) : une ville où un million de personnes pourraient vivre et travailler, avec des bâtiments de 350 mètres de long, et 2000 mètres de hauteur, entourés de forêts synthétiques pour permettre aux bambins de gambader !

Mais un prophète avertit les adorateurs de la télé : «Malheur à la grande ville, lieu de caries purulentes!»

D'autres, par contre, croquent à belles dents les plaisirs de la ville dans les illuminations de la nuit.

9 Et ce spectacle si réussi dans les différents aspects proposés, en particulier par la dynamique des groupes, se termine par une vue d'une beauté fascinante: les tours d'une grande ville dont les fenêtres étincelant de leurs différentes couleurs trouent l'obscurité de la nuit.

\section{Un très beau livre d'images}

Mon plaisir de spectateur vient principalement du fait que je suis constamment surprise, étonnée :

- le décor se transforme à tout moment

- l'occupation volumique de l'espace alterne avec l'apparition de micro-mondes

- le matériau toujours très simple : carton d'emballage, pancartes... permet de traiter toutes sortes de situations

- les acteurs représentent un ensemble social (promoteurs, conseillers d'administration...), mais aussi des individus : une locataire qui trouve tout bien, une autre qui n'accepte pas la différence et va vers la haine de l'étranger, le balayeur qui bosse, celui qui ne fait rien et profite de l'autre

- je pleure avec les expulsés, je ris de la brave dame qui ne sait que sourire niaisement, j'enrage devant le cynisme des banquiers, des promoteurs

- je succombe à l'effet esthétique du final qui s'oppose à tout le reste.

11 Du vrai théâtre. Je ne suis pas déçue, je me régale, j'adhère. Les applaudissements de la salle me confortent dans ce sentiment.

Mais de fait j'adhère à quoi ? Où ai-je été embarquée ? Je fais le point.

Je suis contre les promoteurs, les banquiers, les décideurs en général qui écrasent les petits. Ces petits ne sont pas blancs comme neige non plus ; ils peuvent être racistes, un peu benêts, voire niais, agressifs, égoïstes, sans gêne, mais aussi victimes parce qu'entassés comme des lapins, hyper-réglementés dans leur vie personnelle. Ils n'ont plus qu'à regarder la télé comme des drogués, n'entendant plus, ne voyant plus rien d'autre, soumis qu'ils sont à la parole électronique. Perdant le contact avec la vraie nature, ils foulent l'herbe des yeux, les pieds posés sur leur linoléum.

Et les tagueurs? Qu'en penser? je suis partagée : ils détériorent, mais ils expriment leur malaise.

Et la dernière image, comment l'interpréter ? Fascinante de beauté, la ville lumière 
aspire les êtres. Ville aux tours modernes et dont le cœur en forme de coupole est vieux de mille ans. Ville idéale vers laquelle je dois orienter mes rêves?

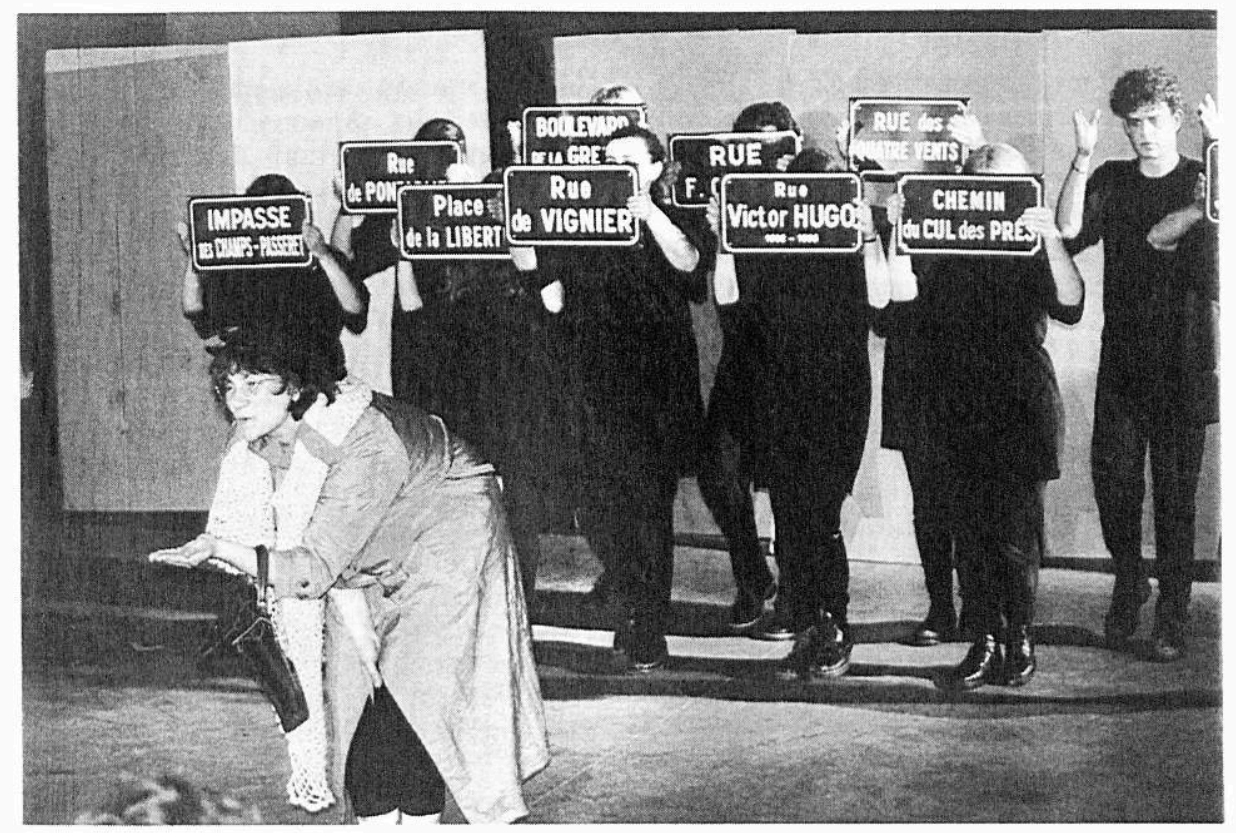

Photo Clément François

12 Et le salut final, avec l'énumération des adresses de chaque étudiant, complétée par une brève indication de leur condition de logement - peu de confort, exiguïté, vue agréable, deux salles de bain ; au cœur de la ville ou à la périphérie, les uns satisfaits, les autres prêts à déménager. Signature d'une enquête qui veut donner l'image de la diversité du tissu urbain : diversité des angoisses, des strates sociales, des individus, des problèmes ; complexité d'un avenir, page blanche pleine d'interrogations que camoufle l'image finale, ô combien esthétique, de la ville du fantasme.

\section{Les quatre murs de la ville}

Un hangar, une estrade noire de plusieurs étages, de décor du spectacle Urbs. Tout, vous saurez tout sur la ville : ses promoteurs, ses bruits, ses constructions, ses règlements, ses occupants, enfin la vie d'une " cité ordinaire».

Beaucoup d'émotions et de dérision dans cette farce sur notre environnement. Les comédiens jouent avec l'espace et le décor très mobile en carton (astucieux). Ils se transforment en murs, en immeubles, en square, en intérieur individuel, mais toujours avec énormément d'imagination. C'est féroce et beau à la fois. C'est décapant et plein de poésie.

Deux moments très forts : la " danse » des feux tricolores, et le défilé des noms de rue, objets symboliques de la cité, traités avec humour et simplicité, très convaincant. La ville nous est dépeinte nue, telle qu'elle est mais aussi telle qu'on voudrait qu'elle soit. Et notre désir devient réalité au tableau final. Les étudiants du TU nous ont communiqué leur énergie. Ils ont fait de leur spectacle non seulement un moment de vif plaisir pour le spectateur mais également une réflexion très profonde sur notre lieu de vie. 

fond de bruitage de bulldozer et de chant maghrébin, les promoteurs chassent les habitants des quartiers pauvres pour en faire sans doute des SDF. Tragique encore cette vieille femme toute tranquille qui donne à manger aux pigeons, prise dans le mouvement infernal des rues qui l'écrasent au sens propre du terme, ou encore ce personnage, témoin oculaire de la folie des bâtisseurs de la plus grande tour du monde, (projet japonais) qui essaie de tirer la sonnette d'alarme des nuisances d'un univers concentrationnaire.

\section{UNE VILLE QUI A BEL AIR}

Devant un mur noir, très haut, sur lequel grimpe une échelle noire, trois praticables imposants en forme d'escaliers, tendus de jupettes noires occupent toute la largeur de la scène. De chaque côté, des cartons d'emballage de grande dimension disposés en paravent ( 3 au sol, 2 à la hauteur du premier praticable) délimitent l'aire de jeu.

fois sont posés presque à plat. Pour ma part, connaissant bien le style du TU, je sais déjà que tous ces éléments cachent des surprises. Ma curiosité est donc dès l'entrée aiguisée, car je sais que mes pronostics d'utilisation de cet espace et de ces cartons vont être détrompés, et je m'en réjouis.

relativement classique encore que les premiers par leur disposition permettent un jeu en cascade dégringolée très dynamique, et que l'échelle malgré sa hauteur est utilisée pour de vrai. Ce n'est pas sans quelques frissons d'inquiétude qu'on regarde garçons et filles escalader cette sorte d'escalier du Bronx. Mais le plus étonnant est l'utilisation au cours du spectacle de ce matériau de forme simple qui se transforme en autant de lieux ou d'objet que nécessite le traitement des thèmes. Ils sont, tour à tour, et dans l'instant : toits de cabanes, tours, métro, appartements HLM, murs à tagger et à cacher (en partie...) l'horreur de la violence, agences immobilières, tribunes, rues, labyrinthes, instruments de voirie pour éboueurs, cabinets d'archives, et enfin sublime cathédrale de toutes les villes du monde, éclairées le temps d'un trop bref instant de la transparence lumineuse des papiers de couleur de leurs minuscules fenêtres en découpage. Ce spectacle, montage de textes qui parlent bien de l'air du temps, où l'on retrouve à côté de Bourdieu, des règlements de copropriété, des enquêtes sociologiques, des discours politiques (récents), des textes de lois (récents), et... des poèmes pour enfants, s'appuie pour faire ressortir l'incongruité sur une théâtralisation qui privilégie une esthétique toujours signifiante et sur des procédés de décalages et de détournement.

Ce qui produit, selon les cas des effets humoristiques, comiques, tragi-comiques ou À l'opposé l'utilisation d'un corps de femme comme " truchement » pour figurer « une ville avec tous ses avantages » dans la scène des agents immobiliers relève plutôt de style chansonnier.

Ailleurs les feux tricolores (des vrais) qui deviennent objets musicaux et qui clignotent sur une agréable musiquette nous invitent à un autre rapport aux choses.

Cette diversité gêne d'autant moins que la mise en scène prend soin au début du spectacle de bien " poser la première pierre » qui est celle des "Causes », ce qui permet ensuite à chacun de naviguer à l'aise parmi les conséquences - mêmes si elles ne sont pas forcément liées entre elles par la logique d'un fil conducteur. Sous les apparences 
d'un jeu proche de l'improvisation chorale ou individuelle se cache une grande rigueur et chaque effet fait mouche - car ça roule sans bavure à tous les niveaux : jeu, régie, lumière et son. Ce qui est dit s'entend, ce qui est suggéré se reçoit. Ici, point n'est besoin d'une technique de jeu sophistiqué, qui affadirait le propos plus qu'elle ne le servirait, en détruisant l'impact de la pétillance des regards, de leur tristesse, ou de leur cruauté, le clin d'œil d'un sourire "béat » (voir la scène des HLM) et la vigueur de l'engagement des corps.

Les 21 acteurs qui déclinent leur véritable identité au salut ne sont pas des comédiens mais des gens comme vous et moi; ils nous disent le monde, à nous spectateurs étonnés, émerveillés de tant d'imaginations jusqu'à nous arracher un oh! de surprise émerveillée devant le bouquet final de ce feu d'artifice qu'est la dernière image : La Cathédrale des Villes Lumières.

\section{Interview du metteur en scène, Joseph Melcore}

\section{QUELLE A ÉTÉ VOTRE MÉTHOdE DE TRAVAIL ?}

La première étape a consisté à se poser la question : Qu'est-ce-que la ville?

Et à lister tout ce que les étudiants disaient : pour moi, la ville c'est... les feux, les lumières de la ville, la violence dans les quartiers, les H.L.M., le métro, la signalétique...

À partir de là nous avons dégagé des thèmes, des objets sur lesquels nous avons improvisé : la ville se déclinait alors en images qu'il fallait ensuite agencer pour trouver le rythme du spectacle.

Il n'y avait donc pas de partition écrite au départ, l'élaboration de la pièce se faisait au fur et à mesure de la participation des étudiants et de leurs apports respectifs en textes divers : règlements intérieurs d'H.L.M.; articles puisés dans des revues scientifiques comme "Sciences et vie »; textes de théâtre; annonces immobilières; poèmes sur la ville; enquêtes sociologiques...

\section{VOUS N'AVEZ DONC PAS VOULU PARLER D'UN SEUL ASPECT DE LA VILLE ?}

Non, bien au contraire! Il s'agissait plutôt de donner aux étudiants la possibilité de parler de tout ce qui les fascinait, les révoltait dans la ville. De la questionner en essayant de faire la lumière et d'éclairer le spectateur.

$\mathrm{Au}$ moment de la création, on écrivait beaucoup sur la violence dans les banlieues et les quartiers. Nous aurions très bien pu monter un spectacle sur ce thème, mais ce n'était pas notre choix : c'est devenu un tableau parmi d'autres dans la pièce.

\section{POURQUOI AVEZ-VOUS PRIS LE PARTI D'UTILISER DES CARTONS ?}

28 L'idée d'utiliser des plaques de carton de $2 \mathrm{~m}$ sur 2 est venue lorsque nous avons voulu restituer l'image des blocs de la ville. En travaillant avec cette matière, d'autres images ont surgi : les cartons des sans logis, la configuration de l'avenue, le métro, les lumières de la ville... Tout cela a contribué à faire du carton un des éléments importants de la pièce.

\section{QUEL EST L'INTÉRÊT DE CE TYPE DE TRAVAIL ?}

29 L'intérêt de ce type de travail consiste dans le fait que chaque étudiant est complètement impliqué dans la création du spectacle et qu'il contribue à l'élaboration de sa propre partition à l'intérieur de la partition globale, même si certains sont parfois 
déroutés par l'incertitude dans laquelle nous nous trouvons durant le voyage. Le scénario de URBS a vu le jour après les premières représentations.

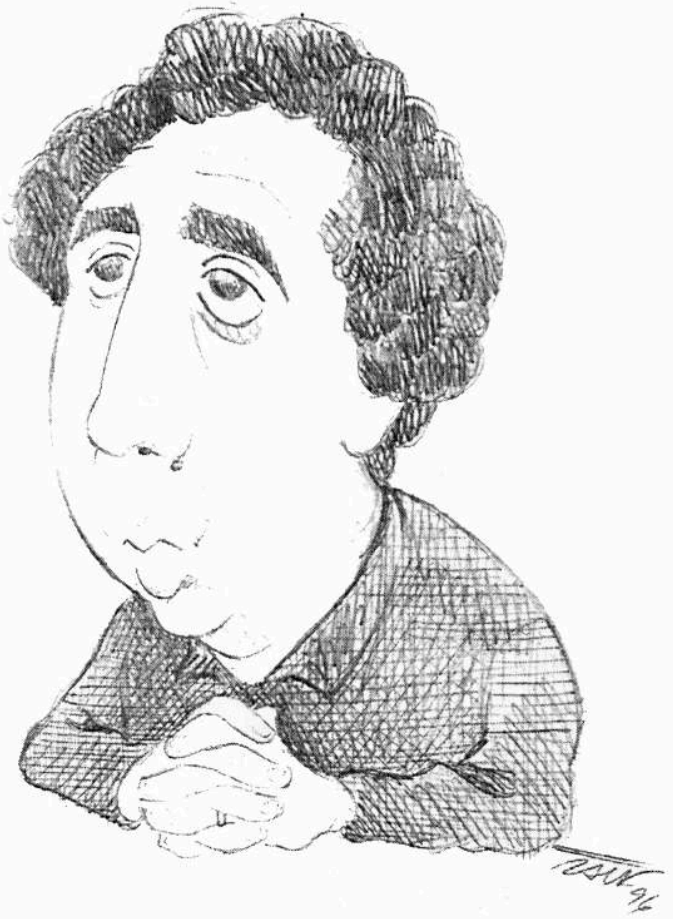

Dessin Raphaël Baud

\section{AUTEUR}

\section{DANY VINGLER}

Adjoint délégué à l'action culturelle 